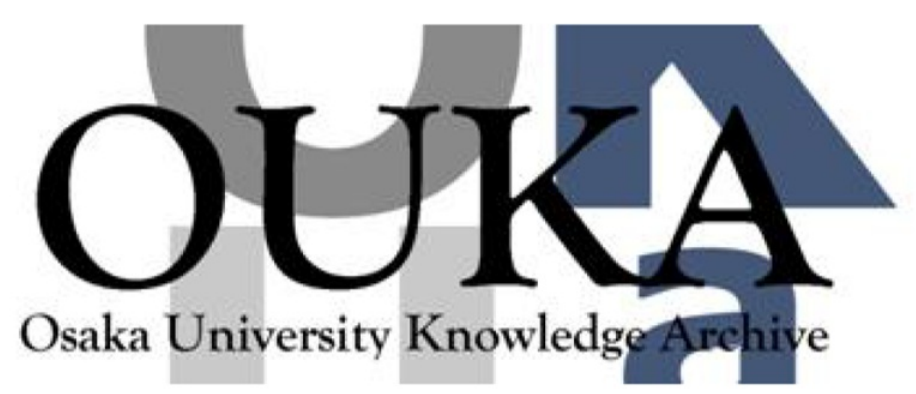

\begin{tabular}{|c|c|}
\hline Title & $\begin{array}{l}\text { Resonance acoustic microbalance with naked- } \\
\text { embedded quartz (RAMNE-Q) biosensor fabricated } \\
\text { by microelectromechanical-system process }\end{array}$ \\
\hline Author(s) & $\begin{array}{l}\text { Kato, Fumihito; Ogi, Hirotsugu; Yanagida, Taiji } \\
\text { et al. }\end{array}$ \\
\hline Citation & $\begin{array}{l}\text { Biosensors and Bioelectronics. 33(1) p. 139- } \\
\text { p. } 145\end{array}$ \\
\hline Issue Date & $2011-12-31$ \\
\hline oaire:version & AM \\
\hline URL & https://hdl. handle. net/11094/84195 \\
\hline rights & $\begin{array}{l}\text { ( } 2011 \text { Elsevier B.V. This manuscript version is } \\
\text { made avai lable under the Creative Commons } \\
\text { Attribut ion-NonCommercial-NoDerivatives } 4.0 \\
\text { International License. }\end{array}$ \\
\hline Note & \\
\hline
\end{tabular}

Osaka University Knowledge Archive : OUKA

https://ir. Library. osaka-u. ac. jp/

Osaka University 


\title{
Resonance Acoustic Microbalance with Naked-Embedded Quartz (RAMNE-Q) Biosensor Fabricated by
} Microelectromechanical-System Process

\author{
Fumihito Kato $^{a}$, Hirotsugu Ogi ${ }^{a, *}$, Taiji Yanagida ${ }^{a}$ \\ Shintaro Nishikawa ${ }^{a}$, Masahiko Hirao ${ }^{a}$, Masayoshi Nishiyama ${ }^{b}$ \\ ${ }^{a}$ Graduate School of Engineering Science, Osaka University \\ Machikaneyama 1-3, Toyonaka, Osaka 560-8531, Japan \\ ${ }^{b}$ Central Workshop, Osaka University \\ Machikaneyama 1-2, Toyonaka, Osaka 560-0043, Japan
}

\begin{abstract}
A resonance acoustic microbalance with a naked-embedded quartz (RAMNE-Q) is realized by a microfabrication method, aiming at broader applications of quartzcrystal microbalance (QCM) biosensors. The RAMNE-Q biosensor consists of three layers; a silicon layer with an engraved microchannel and sandwiching glass layers. The naked AT-cut quartz resonator of 9.3 or $28.5 \mu \mathrm{m}$ thick is located in the microchannel and supported by the silicon micropillors and semicircular walls without fixing, and it is encapsulated by the rigid body. Cupper antennas are used for generating and receiving electromagnetic fields to excite and detect the shear vibration of the quartz oscillator during the solution flow, thereby achieving the noncontact measurement of the resonance frequency. Because of the isolated resonator, the $\mathrm{Q}$
\end{abstract}


factor is high enough (about 1500 at $170-180 \mathrm{MHz}$ ) even in the flowing solution. We succeeded in detecting $1 \mathrm{ng} / \mathrm{ml}$ human immunoglobulin $\mathrm{G}$ in phosphate-bufferedsaline solution via staphylococcus aureus protein A immobilized nonspecifically on the quartz surfaces, demonstrating the high sensitivity and high signal-to-noise ratio of the RAMNE-Q biosensor. It does not require electrodes and is a replacement-free biosensor, and its reusability is confirmed.

Key words: QCM, MEMS, Electrodeless, Replacement-free

\section{Introduction}

It has been recognized that the affinity analysis among various biomolecules is important in broader fields of recombinant-protein-production technology, including molecular-target drug discovery. The labeling methods were intensively used to obtain biochemical information of objective substances selectively. Conventional labeling techniques use large-size tag proteins (e.g. greenfluorescent proteins $(\sim>27 \mathrm{kDa})$, FLAGs with specific antibodies $(\sim>150$ kDa), quantum dots $(\sim>20 \AA)$ ( Bruchez et al. (1998); Green (2004))). These labels, however, cause steric hindrance and then affect the affinity among biomolecules. Furthermore, modification of protein structure for attaching labels will influence biological functions of examining proteins ( Terpe (2003)). These problems accelerate study in developing sufficiently small labels, which would not affect the functions of proteins ( Guignet et al. (2004); Ojida et al. (2006)). Therefore, development of a label-free biosensor without modification of protein structure is significantly important.

Two label-free biosensors are widely recognized. One is the surface-plasmonresonance (SPR) biosensor. The generation condition of surface-plasmon waves 
at metal-dielectric interface is sensitive to the change in the dielectric constant or the refractive index. Adsorption of proteins on the sensor surface varies the effective refractive index, which can be detected through the change in the incident angle for causing SPR ( Kurita et al. (2007); Nedelkov et al. (2007); Hoaa et al. (2007)). However, target molecules near the detection area also cause the change in the refractive index, and the quantitative evaluation of adsorbed molecules is not straightforward. Furthermore, because this method is insensitive to the region outside the evanescent field, it is inapplicable to detection of large molecules and cells, and long aggregation reactions.

The other is the quartz crystal microbalance (QCM) that is a mass-sensitive biosensor; it principally detects the additional mass through decrease in the resonance frequency of the quartz oscillator when objective substances are attached on the surface of the quartz plate in resonance ( O'Sullivan et al. (1999); Hung et al. (2003); Kao et al. (2007); Furusawa et al. (2008); Kao et al. (2009)). This sensor also enables a label-free and real-time measurement of biochemical reaction and can absolutely determine the adsorption quantity of the analyte; it is an ideal biosensor in the field of the drug development and medical diagnostics. However, the conventional QCM biosensors show lower sensitivity than others that use labels, particularly to light-weight molecules. Such an issue is due to the structure of the QCM. When the mass of quartz plate $M$ is large enough compared with the mass of adsorbate $\Delta m$, the resonance frequency shift $\Delta f_{\text {mass }}$ responds as ( Sauerbrey (1959); Eddowes (1987))

$$
\Delta f_{\text {mass }}=-\frac{\Delta m}{M} f_{0}=-\frac{\Delta m_{s} v_{s}}{2 \rho} \cdot \frac{1}{d^{2}}
$$

where $f_{0}$ denotes the original resonance frequency, and $\Delta m_{s}$ is the adsorbed 
mass per unit area (area mass density). $\rho, d$, and $v_{s}$ are the mass density, thickness of the quartz crystal, and shear-wave velocity, respectively. The mass sensitivity of QCM biosensor is then inversely proportional to the square of the thickness; the thinner the quartz plate is, the higher sensitivity the sensor provides ( Ogi et al. (2009a)). Conventional QCM biosensors use AT-cut quartz crystals with resonance frequencies of about 5-10 MHz, corresponding to thicknesses of $166-330 \mu \mathrm{m}$. They have to use Au electrodes for applying effective electric field and for making the gold-thiol reaction to immobilize receptor proteins. However, it has been proven that those electrodes significantly deteriorate the mass sensitivity of QCM due to inertia resistance ( Ogi et al. (2009a)). Furthermore, most QCM biosensors adopted only one side of the quartz plate, remaining the other side exposed to air, for avoiding electric conduction between the electrodes. However, the solvent pressure causes the breakage with thinner quartz plates in this case, restricting the use of thinner quartz resonators.

There are some studies on inverted mesa resonators fabricated by MEMS technology (Abe et al. (2000); Hung et al. (2003); Kao et al. (2007); Kao et al. (2008); Kao et al. (2009)). They used the bottom of the micro cavity formed by ICP (inductively coupled plasma) etching on a quartz substrate as the quartz resonator. The multichannel-type QCM is realized because several micro cavities can be formed on a quartz substrate. However, the operation in the liquid is difficult and the detection area becomes only one side of the quartz resonator. The energy loss due to the structural damping is large because the resonator integrates with the quartz substrate. Forming the cavities by the dry etching method, the limit of the thickness is approximately $20 \mu \mathrm{m}$ due to the large surface roughness, although the quartz plate must be thinner to 
achieve higher sensitivity.

We then developed a wireless-electrodeless method; the electrodeless quartz resonator is operated with the electric field generated by the line antennas to excite and detect the shear vibrations contactlessly ( Ogi et al. (2009a, 2011)). The reaction between biomolecules can be measured quantitatively without any labels and protein modifications by the application of high-frequency blank quartz plates up to $180 \mathrm{MHz}$. This device is capable of pM-order measurement. However, very thin quartz resonators $(\sim 10 \mu \mathrm{m}$ or less $)$ are fragile and easy to break up during the handling to install them in the sensor cell, so that the expert techniques and special tools were required. Also, a part of the quartz plate was fixed by sandwiching with two silicon-rubber gaskets, deteriorating the $\mathrm{Q}$ value ( $~ 800$ at $170 \mathrm{MHz}$ at most).

As a breakthrough to these problems, we proposed the resonance acoustic microbalance with naked embedded quartz (RAMNE-Q) chip (Kato et al. (2011)). It embedded a bare thin quartz resonator in a microchannel fabricated in Si wafer by the microelectromechanical system (MEMS) technology. However, it showed very low extraction rate because of the sticking of the edges of the quartz plate to the channel wall, causing significant leakage of vibration energy. Therefore, only a few number of sensor chips was available by a mere accident. Besides, because of the uneven solution flow, the detection ability was deteriorated. In this study, we significantly improve the microchannel structure to achieve reusable high-frequency (fundamental resonance frequency of $178 \mathrm{MHz}$ ) and high-Q ( 1500) biosensor (see details in the supplemental file). We demonstrate the superior characteristics of the RAMNE-Q biosensor (realtime, label-free, protein-modification free, replacement-free, and quantitative measurement), using specific bindings of human immunoglobulin $\mathrm{G}$ (hIgG) 
with staphylococcal protein A (SPA) and streptococcal protein G (SPG).

In previous wireless-electrodeless QCM biosensors, we used quasistatic electric field along the thickness direction radiated by the line antenna ( Ogi et al. (2009a,b)). Here, we report a new antenna configuration, where a flat rectangle antenna radiates the electromagnetic field and excite the quartz plate with the in-plane electric field. This configuration achieves 10 times amplification of the transduction efficiency compared with the previous method, contributing to more stable baseline. We also investigate the influence of the sodium-chloride concentration on the transduction efficiency. Sodium chloride is widely used in the buffer solutions, but it increases the solution viscosity and tends to shield the electromagnetic wave due to mobile ions. Thus, our new configuration may be affected by the sodium-chloride concentration.

\section{Reusable RAMNE-Q Chip}

Figure 1 shows the structure and appearance of the RAMNE-Q chip that we developed as a breakthrough of the wireless QCM biosensor. It is composed of three substrates (glass-silicon-glass) (Figure 1(a)). The microchannel is constructed in the upper glass substrate and the middle silicon substrate. They include rectangular spaces, where the naked quartz resonator is embedded, and upper and lower surfaces of the quartz plate are supported by the micropillars without fixing (Figures 1(b) and (c)). The in-plane-direction movement of the quartz plate due to the solution flow is restricted by the point supports with the silicon-semicircular humps. The bare AT-cut quartz plate measures $2.5 \mathrm{~mm}$ long, $1.7 \mathrm{~mm}$ wide, and 9.3 or $28.5 \mu \mathrm{m}$ thick. Two glass substrates and silicon substrate are bonded by the anodic bonding method to form a rigid package, 
which protects the fragile thin quartz plate from external forces. Thus, the mechanical contacts to the oscillator are minimized, resulting in nearly free shear vibrations.

[Insert Figure 1 here.]

Because the embedded quartz resonator is electrodeless, the RAMNE-Q chip is free from the inertial resistance of the metal electrodes and can use both surfaces as the active sensing area. Moreover, the structural damping of the vibration is decreased by supporting the resonator by silicon micropillars and semicircular humps without gripper. As a result, the device performs with a high enough Q-factor, even if the quartz resonator is fully immersed in the solution. There is no issue of breakage due to the solution pressure because both surfaces are exposed to the solution. Also, it can improve the restriction of the use in the liquid environment, because the quartz resonator is encapsulated in the rigid package.

The RAMNE-Q is reusable semi-permanently, because the receptor proteins adsorbed nonspecifically on the quartz surfaces can be removed by the washing procedure with a cleaning solution (e.g., piranha solution) flowed in the microchannel. Furthermore, the reassembly is unnecessary, and so it is easy to reuse. By operating at high frequencies $(\sim 180 \mathrm{MHz})$, the device can achieve high sensitivity to the added mass, and the contribution of the viscous damping decreases ( Ogi et al. (2006)). In the previous paper, we demonstrated that the Sauerbrey equation is quantitatively applicable in the case of a highfrequency electrodeless quartz resonator ( Ogi et al. (2009a)).

A micromachining technology is used for the production of the RAMNE-Q chips, so that the mass production by the batch process is possible (Figure S1 
in the supplemental material), and the analysis system combined with other devices such as lab-on-a-chip is also possible. The upper and lower sides of the silicon substrate with the microchannel are bonded with two transparent glass substrates, making it possible to visually observe the quartz surfaces in real time. The details of the device production are shown in the supplemental material (Figure S1).

In the previous wireless QCM system, we used the quasistatic electric field along the thickness direction of the quartz plate by locating two line antennas near one side and a grounding wire near the other side ( Ogi et al. (2009a)). However, the decrease in the perpendicular electric field at the quartz plate was significant with this configuration as the dielectric constant of the solution increased. In this study, we propose an alternative antenna configuration shown in Figure 1(d), where two flat antennas are located so as to sandwich the RAMNE-Q chip. The center line of the cable from the high-power gated amplifier is connected to the upper flat antenna for excitation, and the lower flat antenna is connected toward the center line of the cable to the broadband receiver: The grounding wires are not located near the RAMNE-Q chip. With this configuration, the radiated dynamic electric field principally possesses in-plane components, which will arrive at the quartz oscillator with less attenuation. Indeed, we succeeded in the signal amplification with this method by a factor of 10 compared with the use of previous antenna configuration.

\section{Experimental Section}

hIgG was obtained from Athens Research and Technology, Inc. (No. 16-16090707). SPA was from Zymed Laboratories, Inc. (No. 10-1100). SPG was 
from Cell Sciences (No. CRP154B). All of other chemical substances were purchased from Wako Pure Chemical Industries Ltd.

The RAMNE-Q chip is located in the flow cell made of Teflon shown in Figure 1(d). The solution is flowed into and flowed out of the microchannel through the two pipes. The O-rings are installed to prevent the leakage of the solution at the interface between the RAMNE-Q chip and the flow cell. The flat copper film antennas, which cover the whole quartz plate, are located on the upper and bottom sides of RAMNE-Q chip. The distance from the upper antenna to the quartz surface is $0.34 \mathrm{~mm}$ and that from the lower antennas is $0.35 \mathrm{~mm}$, achieving the noncontacting measurement. Two center lines of two coaxial cables are used for the excitation and signal detection of the quartz resonator through the electromagnetic field. Each core wire of the coaxial cable for the excitation or signal detection of the quartz resonator is connected to each film antenna (for input or output). On the other hand, the grounding line to shield each core line is shunted outside the flow cell. The cell is installed in the shielded box in the homebuilt flow-injection-analysis system, which can continuously measure the resonance frequency shift with the solution flow in the microchannel. The temperature inside the shield box was maintained at $37^{\circ} \mathrm{C}$. The flow rate and pressure of the solution are controlled by the liquid chromatography (System controller and flow unit: SCL-10A, Shimazu, Japan, and Degasser: DGU-20A3, Shimazu, Japan). This system has two injectors, which are connected to the main flow tube before the sensor cell, in order to inject receptor and analyte proteins individually.

The measurement of the resonance frequency is based on the resonance ringdown method that we have originally developed (Hirao et al. (1993)) and is similar to the method used in QCM-D studies (Rodahl et al. (1995); Höök 
et al. (1998)): The tone-burst signals are applied to one of the flat antenna to generate the electromagnetic field, and the thickness shear resonance is excited through the converse piezoelectric effect. After the excitation, the mechanical vibration of the quartz resonator is detected with the other flat antenna through the electromagnetic field radiated as the oscillating electrical potential generated through the piezoelectric effect. The received signals enter a superheterodyne spectrometer, and the amplitude and phase at the same frequency component as the driving signal are recorded. The sampling time for a single measurement is about $10 \mathrm{~ms}$, but we average over 500 measurements, so that the time to acquire one point takes $5 \mathrm{~s}$. The change in the resonance frequency is determined from the phase shift. A series of procedure explained above is managed by a personal computer collectively.

Before all experiments, the washing procedure was executed to remove the proteins and contamination, if any. The piranha-solution $\left(30 \% \mathrm{H}_{2} \mathrm{O}_{2}, 70 \%\right.$ $\mathrm{H}_{2} \mathrm{SO}_{4}$ ) ( Mo et al. (2002); Sivagnanam et al. (2007)) and 2-propanol were injected as cleaning solutions. The ultrapure water was then flowed to rinse the quartz surfaces and microchannel, and activate the naked quartz surfaces. All experiments were performed with a flow rate of $100 \mu \mathrm{l} / \mathrm{min}$.

We first investigate the influence of the sodium-chloride concentration on the transduction efficiency of the RAMNE-Q chip. Sodium chloride has been used in many bioassays as a standard constituent of buffer solutions, and it is important to research its effect on the newly proposed noncontacting method. We measured the change in the peak amplitude of the resonance spectrum varying the sodium-chloride concentration up to $1000 \mathrm{mM}$ in the ultrapure water. 
Next, in order to demonstrate the usefulness of the RAMNE-Q biosensor, we perform a high-speed assay for detecting SPG molecule (21.6 kDa) via hIgG immobilized on both sides of the quartz resonator nonspecifically. In this experiment, the PBS buffer is first flowed in the microchannel to achieve the base-line stability of a few ppm, corresponding to the detectable mass per unit area of $0.01 \mathrm{ng} / \mathrm{mm}^{2}$. hIgG molecules are then immobilized on both sides of the quartz surface nonspecifically by injecting a $200 \mu \mathrm{g} / \mathrm{ml}$ hIgG/PBS solution in the microchannel. After rinsing with the buffer solution, we inject a $1 \mu \mathrm{g} / \mathrm{ml}$ SPG/PBS solution to monitor the specific binding reaction between hIgG and SPG, and then the quartz surfaces were rinsed with the buffer solution flow.

Subsequently, we show the high sensitivity of the RAMNE-Q biosensor through the detection of the hIgG molecules via the SPA immobilized on both sides of the quartz resonator nonspecifically. The PBS is flowed in the microchannel as the buffer solution, and after the baseline becomes stable, a $400 \mu \mathrm{g} / \mathrm{ml}$ SPA/PBS solution is injected to immobilize the SPA molecules nonspecifically on the quartz surfaces. After rinsing the surfaces with the buffer solution, the hIgG solutions with various concentrations are sequentially injected to monitor the binding reactions with immobilized SPA. In this case, a $0.1-\mathrm{M}$ glycin-HCl buffer solution (GHB, pH2.2) and then the PBS solution are injected after each $\mathrm{hIgG}$ solution for dissociating $\mathrm{hIgG}$ from SPA and rinsing the quartz surfaces, respectively.

\section{Results and Discussion}

The Q-factor calculated from the reverberating decay rate was approximately 1500 at the third overtone resonance frequency of $58 \mathrm{MHz}$ quartz resonator 
when the quartz crystal was completely immersed with a solution flow of the flow rate of $100 \mu \mathrm{l} / \mathrm{min}$ in the microchannel. The Q-factor of conventional QCM is reported to be about 2000-3000 (Anderson et al. (2007); Lee et al. (2009)) for quartz resonators of $5-10 \mathrm{MHz}$ in the condition that only one side of the quartz plate is soaked in the liquid, exposing the other side to air. The intrinsic Q-factor usually decreases as the resonance frequency increases because the internal friction of quartz increases with the frequency (Akheiser mechanism) ( Mason et al. (1965)). Also, it decreases remarkably when the entire quartz plate is soaked in the liquid. Taking these effects into consideration, the Q-factor achieved by the RAMNE-Q is significantly high. It actually increased by a factor of 1.8 compared with our previous electrodeless QCM. This result indicates that the structural damping of the RAMNE-Q is lower because of the supports by the silicon micropillars and semicircular walls without fixing. Accordingly, the RAMNE-Q biosensor is expected to perform a high signal-to-noise-ratio measurement.

[Insert Figure 2 here.]

As shown in Figures 2(a) and (b), the resonance-peak amplitudes decrease with the increase of the sodium-chloride concentration in ultrapure water, indicating that the transduction efficiency with the antenna configuration proposed in this study is affected by the ionization degree. This behavior is not dependent on the resonator thickness but on the driving frequency. The charged substrate influences on the ion distribution at the interface between the substrate and solution in the conductive liquid. The surface potential on the substrate attracts ions having the opposite electric charge. On the other hand, the ions having the same electric charge run short. This distribution of the double layer, which is constructed by the surface potential of the substrate and ions 
attracted to it, also occurs on the quartz resonator surface. The conventional QCM with the metal electrodes generates the potential on the quartz surfaces via the piezoelectric effect. The resultant electric field, whose lines originates from the surface and ends on the ground electrode, extends into the conductive liquid. This extent of the electric field induces movement of ions and possible dipole reorientation. As a result, the ions in the electrolyte redistribute to form a very thin double layer (Gouy-Chapman layer) at the interface between the substrate and solution. The more the ion concentration increase, the more the double layer blocks the electric field from penetrating the bulk of the solution (Josse et al. (1992); Yang et al. (1993); Shana et al. (1994)). Because the RAME-Q operation is different from the commercial QCM (the wireless operation of the bare quartz resonator), this theory is inapplicable to the RAMNE-Q biosensor just as it, although the movable ions may affect the amplitude of the quartz resonator because it is common to apply an electric field to the electrolyte. (The quantitative analysis on this effect will be our future work.)

Besides, the solution viscosity increases as the concentration of the sodiumchloride solution increases (Kestin et al. (1981)). With increase in the solution viscosity, the vibration of the quartz resonator is deteriorated by the damping effect due to the resistance in the interface between solution and quartz surface, and therefore the amplitude of the quartz resonator decreases ( Kanazawa and Gordon (1985); Martin et al. (1991); Natesan et al. (2009)). However, the sodium-chloride concentration used in usual biosensor assays are lower than $300 \mathrm{mM}$, and the dependence of excitation efficiency of RAMNE-Q biosensor on the concentration is insignificant in this concentration range as shown in Figure 2(b). Thus, the RAMNE-Q chip and its excitation method 
are applicable to usual biosensor assays.

Figure 3 shows an example of the real-time monitoring of the assay sequence using the RAMNE-Q chip with $178-\mathrm{MHz}$ fundamental resonance frequency. (The quartz thickness is $9.3 \mu \mathrm{m}$.) The hIgG molecules are directly immobilized on the quartz surfaces nonspecifically as the receptor proteins for capturing SPG. When the hIgG solution is switched to the PBS solution, the frequency recovers very slowly, confirming the high affinity of hIgG molecules to the naked quartz surfaces even with the nonspecific binding ( Ogi et al. (2009b)). The final frequency change due to the nonspecific adsorption of $\operatorname{hIg} G$ is 35.0 $\mathrm{kHz}$, corresponding to adsorption of $20.6 \mathrm{ng}$ of hIgG molecules from the Sauerbrey equation. The subsequent injection of the $1-\mu \mathrm{g} / \mathrm{ml} \mathrm{SPG}$ solution caused a rapid decrease in the resonance frequency because of the specific binding between $\mathrm{hIgG}$ and SPG. The amount of the frequency decrease is about $5.0 \mathrm{kHz}$, corresponding to adsorption of $2.94 \mathrm{ng}$ of SPG molecules. The arrival of the PBS solution causes the dissociation reaction. The total assay time for detecting SPG is $2 \mathrm{~h}$, including the immobilization procedure of the receptor protein. This is much shorter than conventional assays with standard QCM and SPR biosensors, which take longer time only for preparing the surface-modified sensor chips before injecting the analyte $(2-3 \mathrm{~h})$. (Washing and rinsing the surface, coating the surface with linkers such as self-assembled monolayers, washing and rinsing the surface, activation of the linker terminals, washing and rinsing the surface, immobilization of the receptor proteins, washing and rinsing the surface).

[Insert Figure 3 here.]

The upper inset in Figure 3 shows the baseline fluctuation of the RAMNE- 
Q biosensor, which is less than $2 \mathrm{ppm}$, corresponding to the mass sensitivity of $0.01 \mathrm{ng} / \mathrm{mm}^{2}$. Thus, the RAMNE-Q is a stable and less-time-consuming biosensor, owing to the high-frequency naked quartz crystal and minimized contacts to the oscillator.

Furthermore, the RAMNE-Q biosensor allows a quantitative assay because of its high frequency nature. Our previous works highly indicate that the massloading contribution to the frequency decrease becomes dominant at frequencies higher than $170 \mathrm{MHz}$ ( Ogi et al. (2009a)), where the adsorbed mass is proportional to the frequency-change ratio. For checking the quantitative capability of the RAMNE-Q biosensor, we estimate the molecular weight of the injected SPG using the experimental result in Figure 3. Assuming the proportionality of the adsorbed mass to the frequency change, we derived the molecular weight of the injected protein based on the reaction-kinetics theory as ( Ogi et al. (2011))

$$
M_{S P G}=M_{h I g G}\left(\frac{\Delta f_{S P G}}{\Delta f_{h I g G}}\right)\left(1-\frac{\alpha_{d}}{\alpha_{a}}\right)^{-1}
$$

Here, $M_{h I g G}$ and $M_{S P G}$ are molecular weights of hIgG and SPG, respectively, and $\Delta f_{h I g G}$ and $\Delta f_{S P G}$ are the amounts of the frequency change caused by adsorption of hIgG and SPG, respectively. $\alpha_{a}$ and $\alpha_{d}$ are exponential coefficients for the binding and dissociating reactions, respectively. We obtain them as $\alpha_{a}=5.87 \times 10^{-3} \mathrm{~s}^{-1}$ and $\alpha_{d}=3.05 \times 10^{-4} \mathrm{~s}^{-1}$ by fitting exponential functions to the frequency changes on binding and dissociating reactions (red lines in Figure 3). Thus, using $M_{h I g G}=150 \mathrm{kDa}, \Delta f_{h I g G}=35 \mathrm{kHz}$, and $\Delta f_{S P G}=5.0 \mathrm{kHz}$, we obtain $M_{S P G}=22.6 \mathrm{kDa}$. This value agrees well with the molecular weight predicted from the amino-acid sequence of $\mathrm{SPG}(=21.6 \mathrm{kDa})$. Because Equation (1) relies on the quantities of adsorbed receptor and target proteins and the 
reaction-kinetics theory, this agreement confirms the quantitative capability and applicability to the affinity evaluation of the RAMNE-Q biosensor.

Figure 4 shows a real-time monitoring for detecting hIgG molecules using nonspecifically immobilized SPA molecules. This measurement involves seven injections of the hIgG solutions with different concentrations $(0.001-10 \mu \mathrm{g} / \mathrm{ml})$. The GHB solution is used for dissociating hIgG from SPA after each binding reaction. The first injection of $10-\mu \mathrm{g} / \mathrm{ml}$ hIgG solution caused the largest frequency decrement, but the injection of the GHB solution after this raised the baseline. The SPA molecules adhere to the quartz surfaces unsystematically with a free posture by flowing the SPA solution, and accumulate threedimensionally on the adsorbed SPA molecule layer. Because the affinity between the accumulated SPA molecules is lower, they were come off the SPA molecules adsorbed directly on the quartz surfaces by the GHB flow together with the hIgG molecules. After this, the number of the SPA molecules on the surface is unchanged, and the frequency decrements are nearly the same in the second to fourth injections of the $10-\mu \mathrm{g} / \mathrm{ml} \mathrm{hIgG}$ solution. The injection of GHB solution cannot completely dissociate hIgG molecules from SPA molecules, and the baseline gradually lowers as the assay is repeated.

[Insert Figure 4 here.]

Therefore, it is unsuitable to evaluate the concentration of the target protein from the frequency decrement. According to the reaction-kinetic theory, the resonant frequency decreases exponentially during the binding reaction, and its exponential coefficient $\alpha$ is proportional to the concentration of the target protein $C_{t}$ as $\alpha=k_{a} C_{t}+k_{d}$, where $k_{a}$ and $k_{d}$ are association and dissociation reaction-velocity constants, respectively ( Liu et al. (2003a)). Thus, it is 
more appropriate to use the $\alpha$ value for evaluating the concentration because it is independent of the amount of the receptor protein on the surface. Figure 5 (a) shows the absolute value of the frequency change during the binding reaction for various concentration hIgG solutions, supporting that the frequency changes exponentially. We showed the maximum frequency change by the dashed line in Figure 5(a) for the injection of $10 \mathrm{ng} / \mathrm{ml}$ hIgG solution when the SPA molecule was not immobilized. This is the case of the negative (nonspecific) binding assay. From Figure 5(a), we can see that the specific biding causes significantly larger frequency change $(\sim 20 \mathrm{kHz}$ for $10 \mathrm{ng} / \mathrm{ml})$ than the nonspecific binding $(0.05 \mathrm{kHz})$, indicating that our RAMNE-Q biosensor possesses the specificity. Even for the lowest concentration analyte $(1 \mathrm{ng} / \mathrm{ml})$, the RAMNE-Q biosensor successfully detects the target protein. Figure 5 (b) shows the $\alpha$ values obtained for different concentration of hIgG solutions. It shows nearly the same value for the same concentration analyte despite different frequency decrement. It shows a correlation with the hIgG concentration as expected. Actually, we used the same RAMNE-Q chip for many measurements and we never dispose of it. The RAMNE-Q chip was washed before the measurement every time. Nevertheless, we obtained identical results as shown with the error bars in Figure 5(b), showing the reproducibility of a single RAMNE-Q biosensor.

[Insert Figure 5 here.]

We also evaluate the binding affinity from the binding curves. Because the equilibrium constant $K_{A}$ is given by $K_{A}=k_{a} / k_{d}$ ( Liu et al. (2003a,b)), we can determine these constants by determining the $\alpha$ value for various concentration hIgG solutions (Figure $5(\mathrm{~b})$ ). The results are shown in Table 1 together with previously reported values, showing agreements in reasonable range. 
These results indicate that the newly developed RAMNE-Q biosensor has the ability to measure the amount of mass adsorption quantitatively in high resolution with high sensitivity and monitor binding reactions in real time without any labeling. Moreover, the quartz plate and microchannel can be reused repeatedly many times for the next experiment by washing and rinsing them with the strong acid solution and the ultrapure water flowed in the microchannel, respectively.

\section{Conclusions}

We have developed the resonant acoustic microbalance with naked embedded quartz (RAMNE-Q) chips fabricated by the MEMS process, which can be operated by the flat antennas contactlessly, washed with a strong acid solution flowed in the microchannel, and therefore reused semi-permanently. The thin quartz resonator $(9.3 \mu \mathrm{m}$ thick) was supported by the micropillars and semicircular walls without fixing in the inner space of the microchannel. The Q-factor of the RAMNE-Q sensor in the buffer-solution flow was high enough (1500) to achieve a stable baseline of the frequency fluctuation $(<\sim 2 \mathrm{ppm}$ and the mass sensitivity of $0.01 \mathrm{ng} / \mathrm{mm}^{2}$ ).

The influence of the ionization degree of solution on the transduction efficiency for vibration of the built-in quartz resonator in the RAMNE-Q was investigated by varying the concentration of the sodium chloride, and it was shown that the deterioration of the vibrational amplitude was insignificant in usual concentration range of the sodium chloride at the biosensor assay.

It was then shown that high-speed analysis and reusable assay were possible 
with the RAMNE-Q chip through sequential biosensor procedure (nonspecific adsorption by injection of the receptor protein and binding reaction by injecting analyte).

We demonstrated that the device was well capable of detecting the frequency change caused by the capture of hIgG via SPA immobilized on the quartz surfaces nonspecifically, with high sensitivity and in real time without any labeling and protein-modification procedures. We succeeded in the detection of $1 \mathrm{ng} / \mathrm{ml}$ hIgG via SPA immobilized nonspecifically.

The affinity of the hIgG for the SPA was examined from the monitoring results, and it was shown that the observed reaction was caused by the specific binding. Also, the estimated molecular weight of SPG molecule from the frequency responses agreed well with the theoretical value. These results highly confirm that the RAMNE-Q biosensor is applicable to the affinity assay. Further thinning of the quartz plate will be achieved because of the systematic MEMS process, which will be our future work, opening a new door of the ultrahigh-sensing technology in the life science.

\section{Acknowledgement}

The quartz plates divided into rectangular form were supplied with the courtesy of Dr. Koyama of Nihon Dempa Kogyo Co., Ltd. 


\section{Appendix A. Supplementary data}

Supplementary data associated with this article can be found, in the online version, at doi:. 


\section{References}

Abe, T. and Esashi, M. Sens. Actuators A. 2000, 82, 139-143.

Anderson, H.; Jonsson, M.; Vestling, L.; Lindberg, U.; Aastrup, T. Sens. Actuators B. 2007, 123, 27-34.

Bruchez, M.; Moronne, M.; Gin, P.; Weiss, S.; Alivisatos, A. Science 1998, 281, 2013-2016.

Eddowes, M. J. Biosensors 1987, 3, 1-15.

Furusawa, H.; Takano, H.; Okahata, Y. Anal. Chem. 2008, 80, 1005-1011.

Green, M. Angew. Chem., Int. Ed. 2004, 43, 4129-4131.

Guignet, E.; Hovius, R.; Vogel, H. Nat. Biotechnol. 2004, 22, 440-444.

Hirao, M.; Ogi, H.; Fukuoka, H. Rev. Sci. Instrum. 1993, 64, 3198-3205.

Hoaa, X.; Kirk, A.; Tabrizian, M. Biosens. Bioelectron. 2007, 23, 151-160.

Höök, F.; Rodahl, M.; Brzezinski, P.; Kasemo, B. Langmuir 1998, 14, 729-734.

Hung, V. N.; Abe, T.; Minh, P. N.; Esashi, M. Sens. Actuators A. 2003, 108, 91-96.

Josse, F.; Shana, Z. A.; Haworth, D. T.; Licw, S.; Grunzc, M. Sens. Actuators B, 1992, 9, 97-112.

Kanazawa, K.; Gordon, J. Anal. Chem. 1985, 57, 1770-1771.

Kao, P.; Goyal, A.; Allara, D.; Tadigadapa, S. Proc. Transducers' 07, 2007, 2311-2314.

Kao, P.; Patwardhan, A; Allara D.; Tadigadapa, S. Anal. Chem. 2008, 80, $5930-5936$.

Kao, P.; Allara, D.; Tadigadapa, D. Sens. Actuators B. 2009, 142, 406-411.

Kato, F.; Ogi, H.; Yanagida, T.; Nishikawa, S.; Nishiyama, M.; Hirao, H. Jpn. J. Appl.Phys. 2011, 50, 07HD03.

Kestin, J.; Khalifa, H. E.; Correia, R. J. J. Phys. Chem. Ref. Data. 1981, 10, 
$71-87$.

Kurita, R.; Yokota, Y.; Ueda, A.; Niwa, O. Anal. Chem. 2007, 79, 9572-9576.

Lee, D.; Yoo, M.; Seo, H.; Tak, Y.; Kim, W.; Yong, K.; Rhee, S.; Jeon, S. Sens. Actuators B. 2009, 135, 444-448.

Liu, C.Y.; Yu, X.; Zhao, R.; Shangguan, D.; Bo, Z.; Liu, G. Biosens. Bioelectron. 2003, 18, 1419-1427.

Liu, C.Y.; Yu, X.; Zhao, R.; Shangguan, D.; Bo, Z.; Liu, G. Biosens. Bioelectron. 2003, 19, 9-19.

Martin, S.; Granstaff, V.; Frye, G. Anal. Chem. 1991, 63, 2272-2281.

Mason, W. in Physical Acoustics, edited by W. Mason, Academic, New York, 1965, Vol.IIIB, p.254.

Mo, X.T.; Zhou, Y.P.; Lei, H.; Deng, L. Enzyme Microbial. Technol. 2002, 30, $583-589$

Natesan, M.; Cooper, M. A.; Tran, J. P.; Rivera, V. R.; Poli, M. A. Anal. Chem. 2009, 81, 3896-3902.

Nedelkov, D. Anal. Chem. 2007, 79, 5987-5990.

Ogi, H.; Motohisa, K.; Hatanaka, K.; Hirao, M. Anal. Chem. 2006, 78, 69036909.

Ogi, H.; Nagai, H.; Fukunishi, Y.; Hirao, M.; Nishiyama, M. Anal. Chem. 2009a, 81, 8068-8073.

Ogi, H.; Fukunishi, Y.; Nagai, H.; Okamoto, K.; Hirao, M.; Nishiyama, M. Biosens. Bioelectron. 2009b, 24, 3148-3152.

Ogi, H.; Yanagida, Y.; Hirao, M.; Nishiyama, M. Biosen. Bioelectron. 2011, $26,4819-4822$.

Ojida, A.; Honda, K.; Shinmi, D.; Kiyonaka, S.; Mori, Y.; Hamachi, I. J. Am. Chem. Soc. 2006, 128, 10452-10459.

O’Sullivan, C. K.; Guilbault, G.G. Biosens. Bioelectron. 1999, 14, 663-670. 
Rodahl, M.; Höök, F.; Krozer, A.; Brzezinski, P.; Kasemo, B. Rev. Sci. Instrum. 1995, 66, 3924-3930.

K, Saha.; F, Bender.; E, Gizeli. Anal. Chem. 2003, 75, 835-842.

Shana, Z. A. and Josse, F. Anal. Chem. 1994, 66, 1955-1964.

Sauerbrey, G. Z. Z. Phys. 1959, 155, 206-222.

Sivagnanam, V.; Sayah, A.; Vandevyver, C.; Gijs, M.A.M. Proc. Transducers' 07, 2007, 525-528.

Terpe, K. Appl. Microbiol. Biotechnol. 2003, 60, 523-533.

Yang, M.; Thompson, M. Anal. Chcm.1993, 65, 3591-3597. 


\section{Table}

Table 1

The thermodynamic constants determined from reaction curves on differenceconcentration hIgG solutions.

\begin{tabular}{cccc}
\hline \hline & $k_{a}\left(\mathrm{M}^{-1} \mathrm{~s}^{-1}\right)$ & $k_{d}\left(\mathrm{~s}^{-1}\right)$ & $K_{A}\left(\mathrm{M}^{-1}\right)$ \\
\hline Present & $1.52 \times 10^{4}$ & $5.84 \times 10^{-4}$ & $2.59 \times 10^{7}$ \\
Saha et al. (2003) & $0.80 \times 10^{4}$ & $2.77 \times 10^{-4}$ & $2.90 \times 10^{7}$ \\
Ogi et al. (2006) & $2.37 \times 10^{4}$ & $4.56 \times 10^{-4}$ & $5.21 \times 10^{7}$ \\
\hline \hline
\end{tabular}




\section{Figure Captions}

Fig. 1 (Color) (a)Stack of the RAMNE-Q chip. (b) The rectangular space in the silicon microchannel, where the quartz resonator is installed. (c) Crosssection A-A of Figure 1(b). The quartz plate is supported by the micropillars and semicircular wall without fixing. (d) Schematic cross section of the specialized cell, where the RAMNE-Q chip with the copper film antennas is located. The two pipes for the introduction and exhaust of the solution are attached.

Fig. 2 (a) Deterioration of the resonance amplitude with increasing the concentration of the sodium chloride in ultrapure water. (b) Relationship between the sodium-chloride concentration and the resonance-peak amplitudes at the fundamental resonance frequency of $178 \mathrm{MHz}$ quartz resonator and the third over tone of $58 \mathrm{MHz}$ quartz resonator.

Fig. 3 Real-time monitoring of the frequency change caused by the injection sequence measured by the RAMNE-Q biosensor. The fundamental resonance frequency is $178 \mathrm{MHz}$. Arrows indicate the arrival times of the corresponding solutions at the RAMNE-Q chip. Insets enlarge the frequency changes. The red lines are fitted exponential functions for determining the exponential coefficients.

Fig. 4 (Color) As-measured frequency response for an injection sequence. Arrows indicate the arrival times of solutions at the RAMNE-Q chip.

Fig. 5 (Color) (a) Binding curves for injections of hIgG/PBS solutions of various concentrations from $1 \mathrm{ng} / \mathrm{ml}$ and $10 \mu \mathrm{g} / \mathrm{ml}$. Note that a logarithmic scale is used in (a). (b) The exponential coefficient $\alpha$ obtained by fitting the exponential function to the binding curve for various concentration hIgG solutions. 

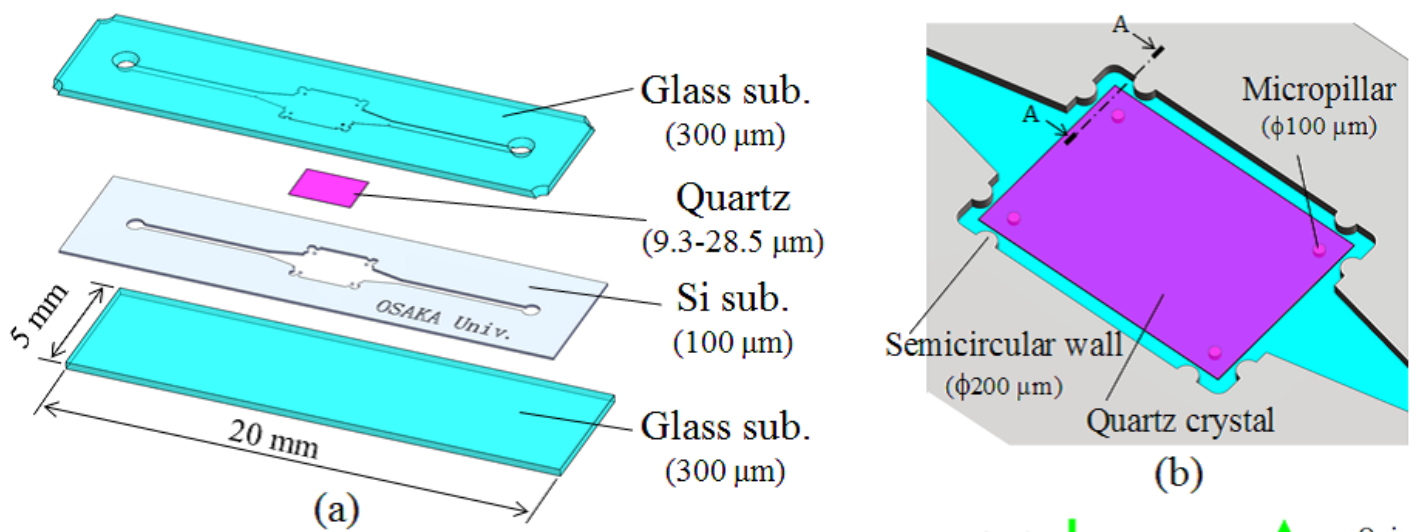

(b)

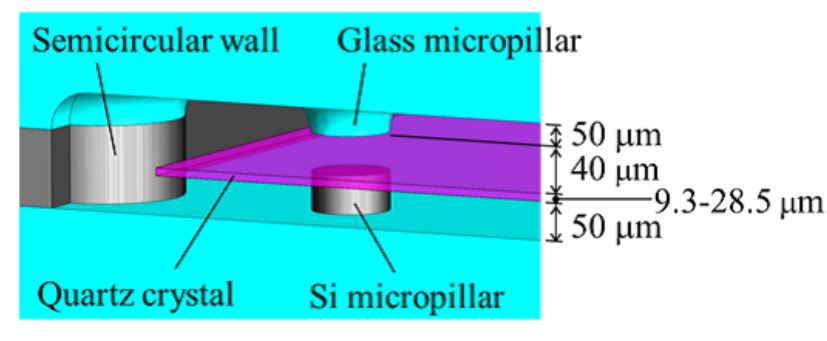

(c)

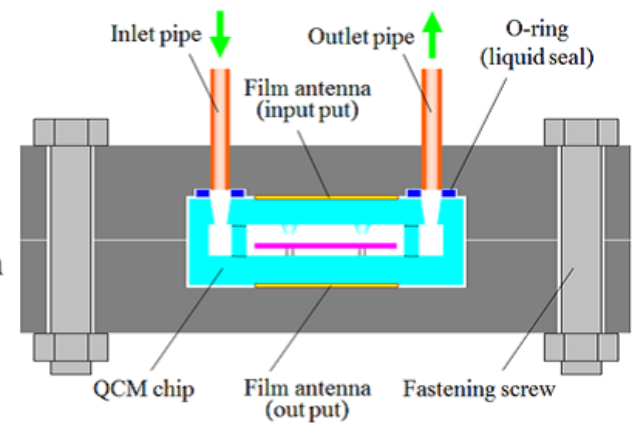

(d)

Fig. 1. 

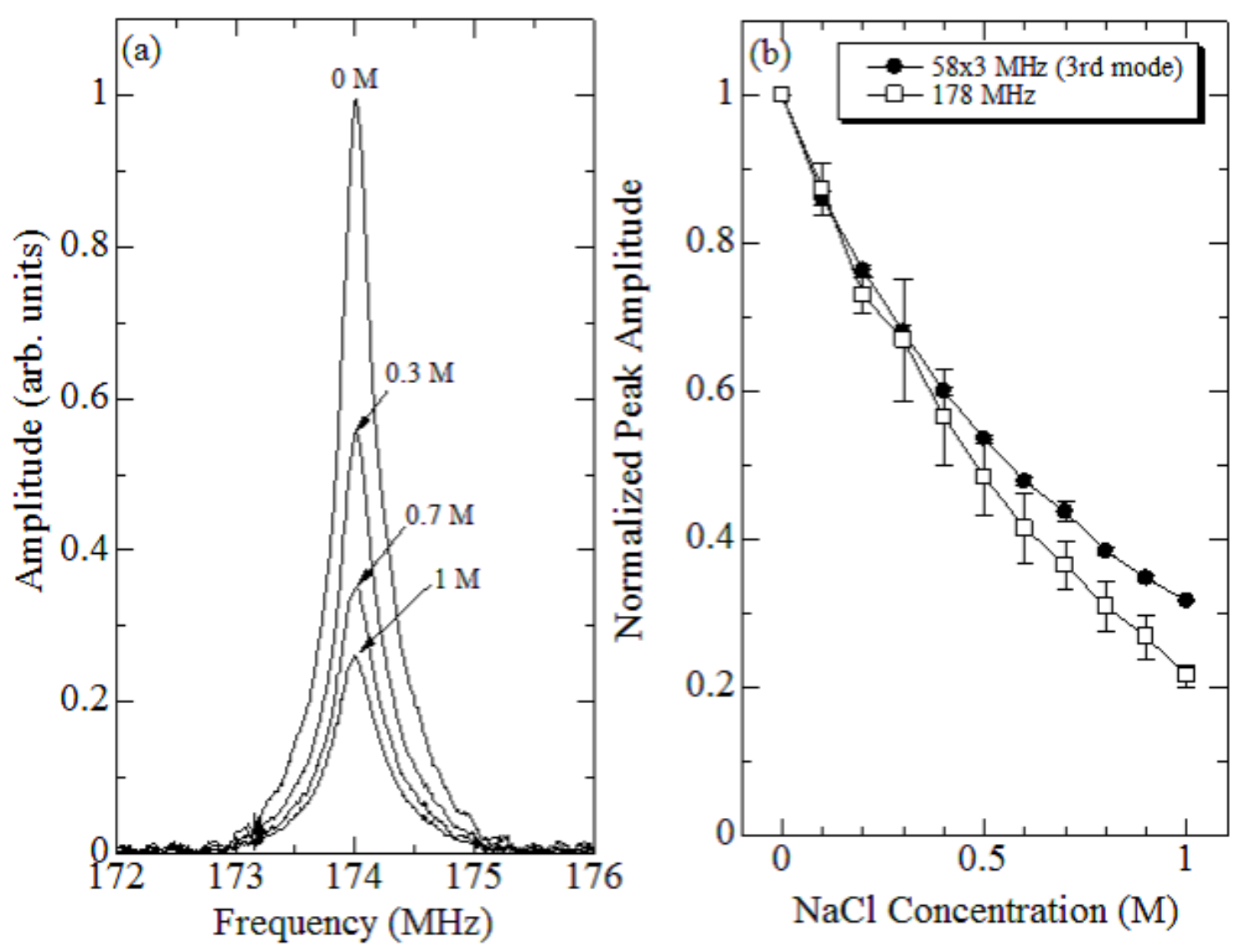

Fig. 2. 


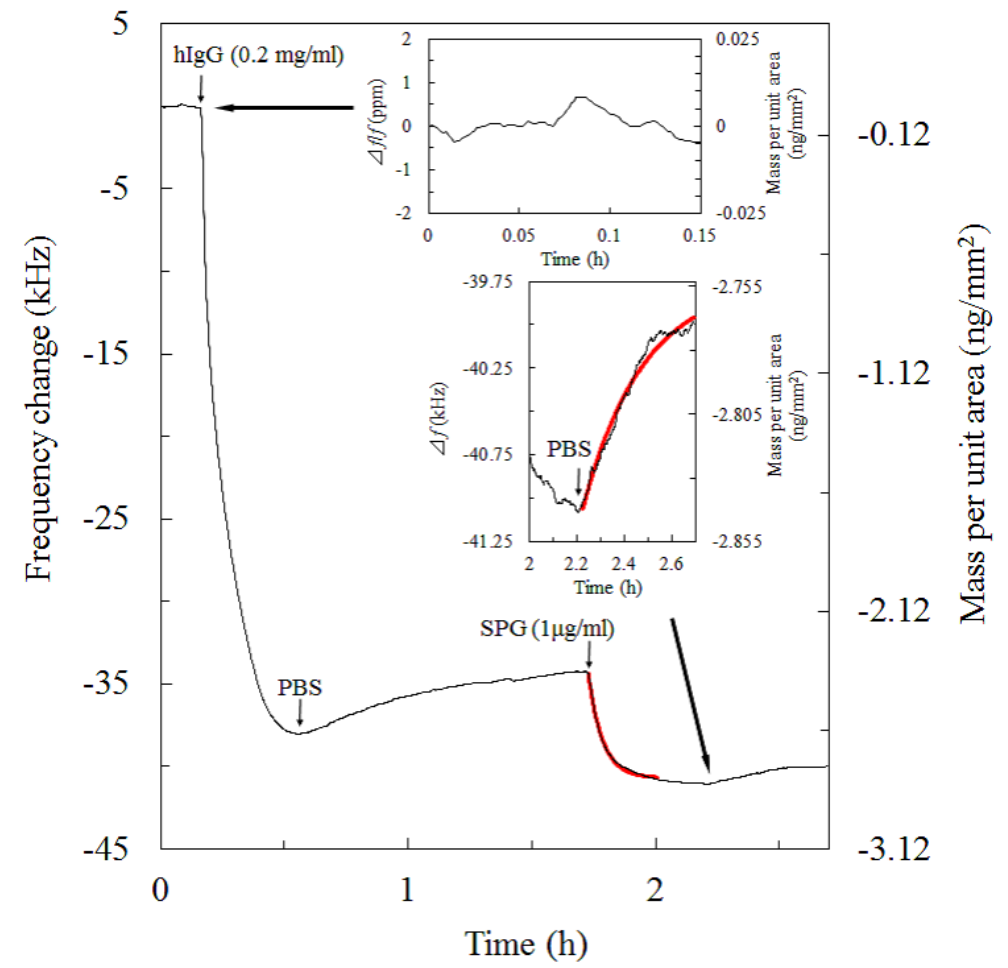

Fig. 3. 


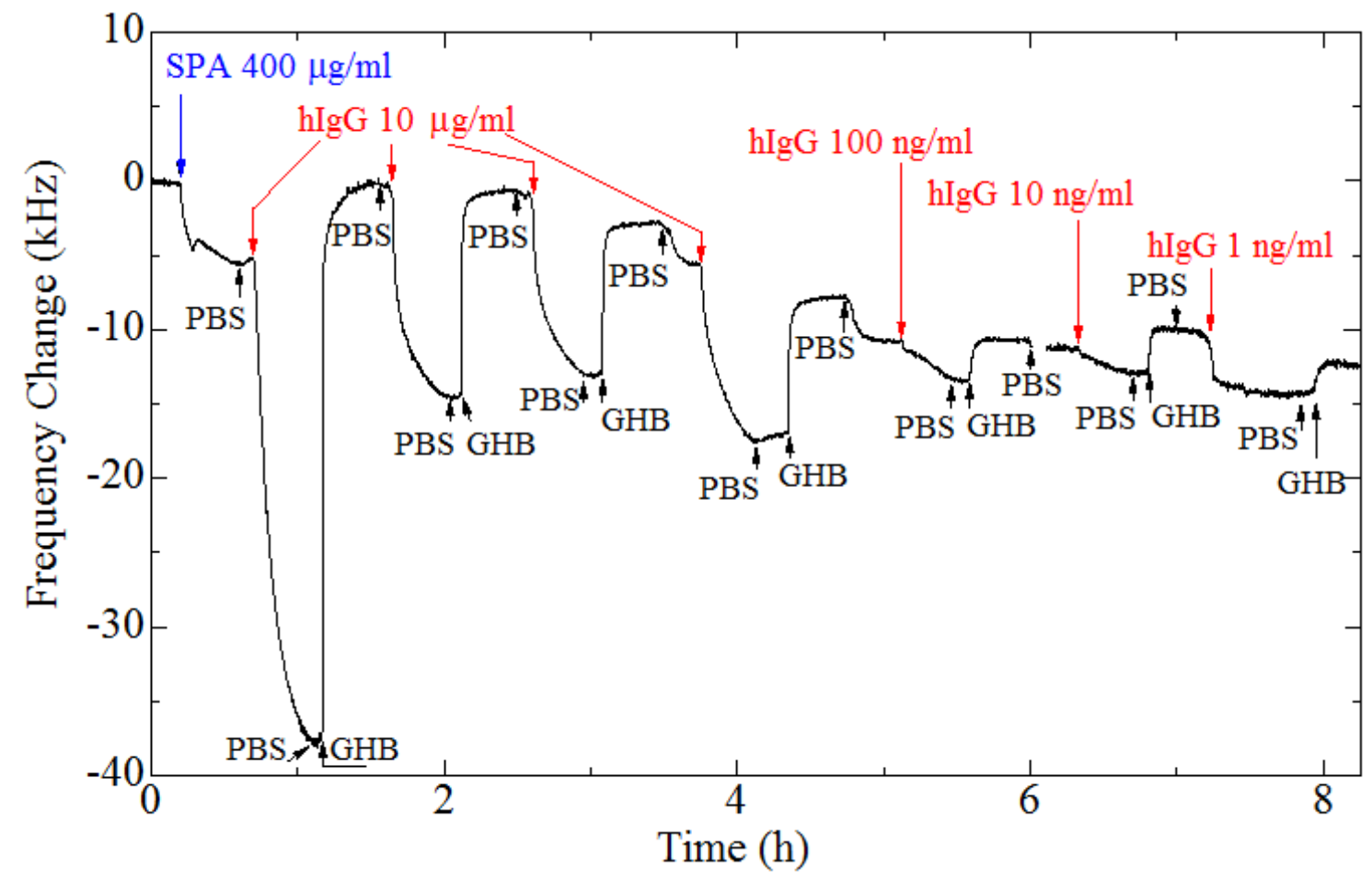

Fig. 4. 

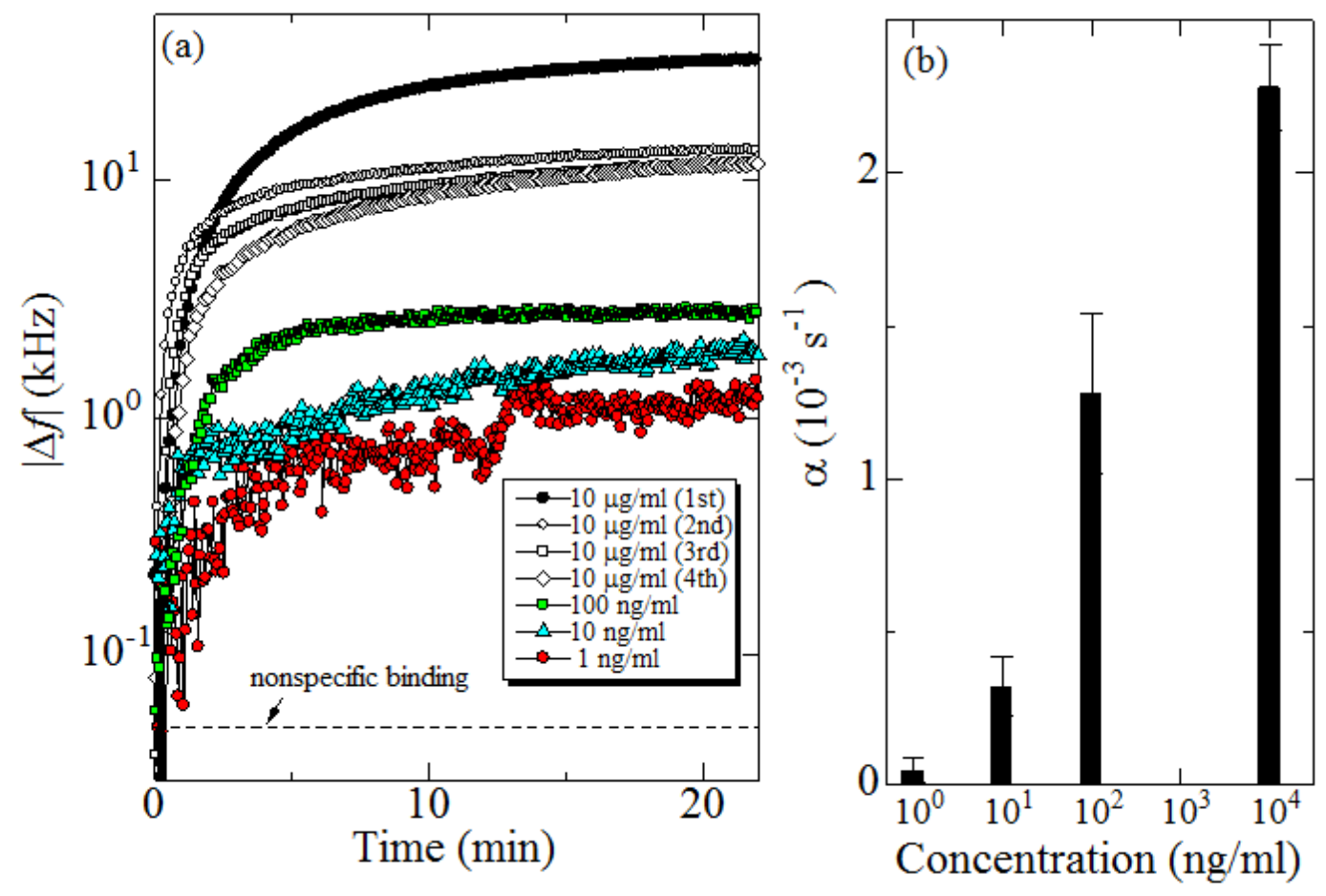

Fig. 5 . 